\title{
Geometry based Dynamic Modeling of the Neuron-Electrode Interface
}

\begin{abstract}
A dynamic model of the neuron-electrode interface is presented which is based on the interface geometry and the electrical properties of the neuronal membrane. The model is used to compute the potential at the electrode and the local membrane potentials. Extracellular as well as intracellular current stimulation can be simulated.

The results demonstrate that extracellular recorded action potentials with several shapes and amplitudes can be produced, depending on the electrical properties of the interface and the neuronal membrane. With homogeneous membrane properties, only small amplitudes are simulated. High amplitudes are produced with decreased concentration of voltage sensitive channels in the lower membrane. Resemblance of the shape of the intracellular potential is accomplished by decreasing the capacity of the lower membrane.
\end{abstract}

J.R. Buitenweg ${ }^{1}$, W.L.C. Rutten ${ }^{1}$ and E. Marani ${ }^{1,2}$

\section{I. : INTRODUCTION}

A well defined electrical contact between a cultured neuron and a substrate embedded microelectrode is important for extracellular recording as well as stimulation. Basically, this contact is mediated by the volume conducting properties of the extracellular space: the neuron-electrode interface. When a neuron covers, or seals, an electrode completely, a specific part of the interface becomes important for the neuron-electrode contact: the sealing gap between the neuronal membrane and the substrate. Experimental results show that in this case the recorded action potentials increase in amplitude from tens of microvolts up to a few millivolts and sometimes even resemble the shape of the intracellular potential. Furthermore, the stimulation threshold decreases $[1,2]$.

These improvements are primarily subscribed to the resistance of the sealing gap. However, it is also recognized that the high amplitudes of extracellular recordings and, even more, resemblance to the shape of the intracellular potential can not solely be explained by a high resistance of the sealing gap. Additional assumptions are required, such as altered ionic conductivities of the sealing part of the membrane, which indicates a secondary effect of sealing $[1,2,3]$.

These effects of sealing will depend on the geometry of the neuron-electrode interface, i.e. the size, shape and mutual position of neuron and electrode. From previous

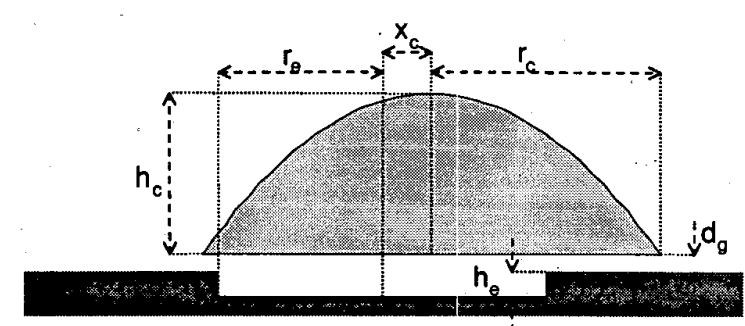

Fig. I Parametrical geometry of the neuron-electrode interface.

work, it was demonstrated that finite element modeling is a proper way to link the electrical behavior of the interface to its geometry, since it inherently accounts for the current that flows from the sealing gap through the neuronal membrane [4]. Therefore, this method is applied again in order to provide a tool for studying the effects of sealing on the electrical properties of the lower membrane and the consequences for the total electrical behavior of the neuron-electrode interface.

The model, presented in this paper, dynamically computes the local transmembrane potentials and currents and also the potential at the surface of the embedded microelectrode. The interface geometry, the conductivity of the sealing gap, the local membrane properties and the intracellular or the extracellular stimulation currents are all included in the model.

\section{METHODS}

The geometry of the neuron-electrode interface is depicted in fig. 1. The neuron is modeled as a circular soma of radius $r_{c}=20 \mu \mathrm{m}$, with a parabolic profile (height $\left.h_{c}=10 \mu \mathrm{m}\right)$. The axon and dendrites of the neuron are not included in this version of the model, since we are primarily interested in situations in which neurons cover electrodes completely or partially. Therefore, the neuron is positioned with an eccentricity, $x_{c}$, on top of an

\footnotetext{
TInstitute for Biomedical Technology

Faculty of Electrical Engineering

University of Twente,P.O. Box 217, 7500 AE Enschede,

The Netherlands

Email: J.R.Buitenweg@el.utwente.nl

${ }^{2}$ Neuroregulation Group

Dept. of Neurosurgery,

Leiden University Medical Centre

The Netherlands
} 
electrode with radius $r_{e}=5 \mu \mathrm{m}$. In this paper the neuron is centered on the electrode, so $x_{c}=0$. The electrode is located in the bottom of a well with a depth of $h_{e}=0.8 \mu \mathrm{m}$. A sealing gap of thickness $d_{g}=10 \mathrm{~nm}$ is modeled between the soma and the substrate.

The geometry of the neuron-electrode interface is implemented in ANSYS Finite Element Software (SAS IP inc. Houston) release 5.5. The medium surrounding the interface is represented by a volume conductor of conductivity $\sigma_{\text {medium }}=1.65 \mathrm{~S} / \mathrm{m}$. This volume is meshed and filled with tetrahedral shaped volume elements (SOLID98) which permit numerical solutions of the Poisson equation:

$$
\vec{\nabla} \cdot(\sigma \vec{\nabla} V)=0
$$

with $\mathrm{V}$ the electrical potential and $\sigma$ the conductivity of the medium. The size of the elements varied from $0.5 \mu \mathrm{m}$ in the sealing gap to several microns far away from the neuron-electrode interface.

The nodes at the outer boundary of the modeled part of the culture medium are set to zero potential, representing a counter electrode which is far away from the neuronelectrode interface. All nodes on the surface of the electrode are coupled in the voltage domain to a single node, which represents the entire electrode surface. No electrical properties of the electrode-electrolyte interface are implemented in this version of the model.

Using the finite element method, a static relationship is formulated between the potentials, $\vec{u}_{m}$, at the membrane nodes, the potential, $u_{e}$, at the node representing electrode surface and the currents into these nodes, $\vec{i}_{m}$ and $i_{e}$ respectively.

$$
K_{s} \cdot\left[\begin{array}{l}
\vec{u}_{m} \\
u_{e} \\
u_{i}
\end{array}\right]=\left[\begin{array}{l}
\vec{i}_{m} \\
i_{e} \\
i_{i}
\end{array}\right]
$$

In this relationship, all membrane potentials are defined with respect to the intracellular potential $u_{i}$, which is represented by a single node, separate from the extracellular volume conductor. Intracellular stimulation can be applied by injecting a current $i_{i}$ into this node.

When the local membrane potentials and the currents injected to the electrode and the intracellular node are known, the local membrane currents follow from

$$
\vec{i}_{m}=K_{m m} \cdot \vec{u}_{m}+K_{m i} \cdot\left[\begin{array}{l}
i_{e} \\
i_{i}
\end{array}\right]
$$

with $K_{m m}$ and $K_{m i}$ derived from $K_{s}$. Furthermore, the electrode potential and intracellular potential are computed as

$$
\left[\begin{array}{l}
u_{e} \\
u_{i}
\end{array}\right]=K_{m e i} \cdot \vec{u}_{m}+K_{i e i} \cdot\left[\begin{array}{l}
i_{e} \\
i_{i}
\end{array}\right]
$$

with $K_{m e i}$ and $K_{i e i}$ derived from $K_{s}$.

The dynamic part of the model consists of the time and voltage dependent properties of the neuronal membrane. The time derivatives of all nodal membrane potentials and channel activation constants are computed from the nodal membrane potentials and currents, according to the model of the chick dorsal root ganglion neuron [3]:

$$
\begin{aligned}
\frac{d u_{m}}{d t} & =\frac{1}{C_{m}}\left[i_{m}-\hat{g}_{N o} m^{3} h\left(u_{m}-u_{N a}\right)-\hat{g}_{K} n^{4}\left(u_{m}-u_{K}\right)-\hat{g}_{C l}\left(u_{m}-u_{C l}\right)\right] \\
\frac{d h}{d t} & =\frac{\lambda_{h}\left(h_{\infty}\left(u_{m}\right)-h\right)}{\tau_{h}\left(u_{m}\right)} \\
\frac{d n}{d t} & =\frac{\lambda_{n}\left(n_{\infty}\left(u_{m}\right)-n\right)}{\tau_{n}\left(u_{m}\right)}
\end{aligned}
$$

with $h$ and $n$ the $\mathrm{Na}^{+}$inactivation and $\mathrm{K}^{+}$activation constants. The $\mathrm{Na}^{+}$activation constant $m$ is considered to be very fast and therefore computed directly from $u_{m}$. The membrane capacity $C_{m}$ and ion specific conductances are computed using the membrane area that is represented by each node.

Simulations are focussed on the influence of membrane properties of the lower membrane on extracellular recording. Firstly, a simulation is performed with stimulus amplitudes resulting in sub- and supra threshold depolarization's of the lower membrane. Secondly, a number of simulations is performed with intracellular stimulation in which the properties of the lower membrane are altered.

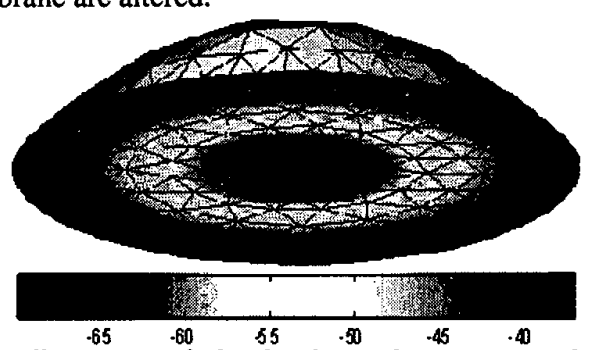

fig.2 Illustration of the local membrane potentials, as computed from extracellular stimulation with a current pulse of $-2.5 \mathrm{nA}$. The cell is seen from below and the depolarized part of the membrane covers the electrode (not shown). In the sealing part of the membrane, the depolarization changes radially towards an hyperpolarization equal to the upper membrane. The resting membrane potential is $-65 \mathrm{mV}$. 


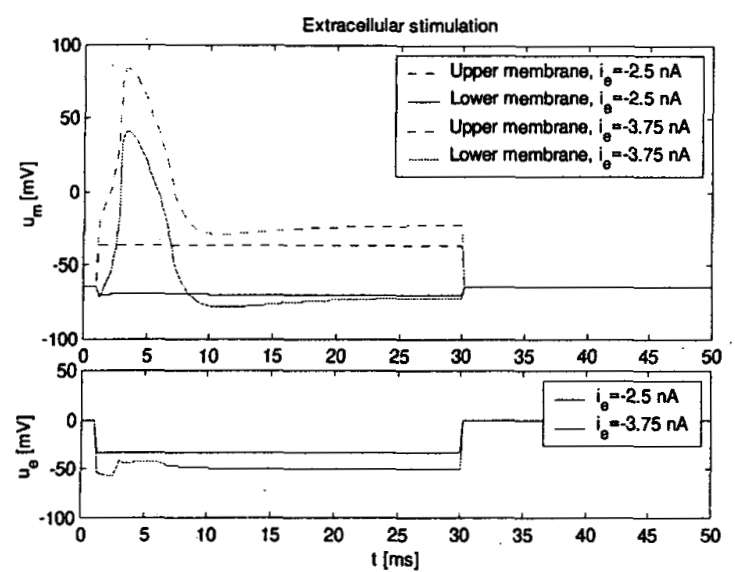

(a)

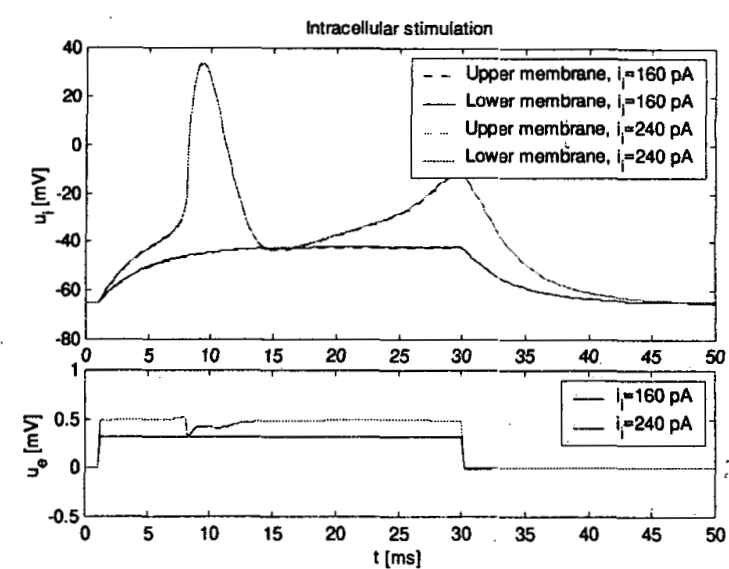

(b)

fig. 3 (a) Extracellular current stimulation. The lower membrane depolarizes, while the upper membrane is hyperpolarized. An action potential is initiated at a stimulus amplitude of $-3.75 \mathrm{nA}$ and this action potential is recorded extracellularly. (b) Intracellular current stimulation. The total membrane depolarizes with the membrane time constant. The initiated action potential can be recorded extracellularly, but has a low amplitude.

\section{RESULTS}

In the first simulation, an extracellular stimulation current of $-2.5 \mathrm{nA}$ is applied during $29 \mathrm{~ms}$. The negative sign of this current indicates that the current flow is directed towards the electrode. As an illustration, the computed local membrane potentials are depicted in fig. 2. In fig. $3 \mathrm{a}$, the potentials of nodes at the upper and lower membrane are plotted versus time, together with the results of a simulation with a stimulus current of -3.75 $n A$. The upper diagram shows the local membrane potentials at an upper and a lower membrane node versus time. At a stimulus current of $-2.5 \mathrm{nA}$, the lower membrane is depolarized but not sufficiently to generate an action potential. The upper membrane is hyperpolarized, but the change in potential is smaller than the lower membrane potential. The time constant of this depolarization is very low, since the membrane capacity is in parallel with the resistance of the sealing gap (which is $13.3 \mathrm{M} \Omega$ ). At the electrode (lower diagram), a 'passive' response occurs due to electrode current and sealing resistance. At a stimulus current of $-3.75 \mathrm{nA}$, a supra threshold depolarization of the lower membrane occurs, resulting in an action potential. The upper membrane also depolarizes after initial hyperpolarization, driven by the inward $\mathrm{Na}^{+}$current through the lower membrane (not shown). On the passive response of the electrode, an active response with an amplitude of $10 \mathrm{mV}$ is superponed due to the current through the lower membrane.

In fig. $3 \mathrm{~b}$, the results of two simulations with intracellular stimulation currents of $160 \mathrm{pA}$ and $240 \mathrm{pA}$ during $29 \mathrm{~ms}$ are depicted. Different from extracellular stimulation, the depolarization of the membrane has a time constant of $3.6 \mathrm{mS}$, which is the membrane time constant. Also, the upper and lower membrane potentials only differ very slightly. This difference is due to the potential drop over the sealing gap which occurs as a result of the outward current through the lower membrane, as can be seen from comparison with the electrode potential. At a stimulus current of $240 \mathrm{pA}$ the membrane depolarization is supra threshold and an action potential develops. Due to this action potential, an active response of approximately $100 \mu \mathrm{V}$ is superponed on the passive response of the electrode potential.

In fig. $4 \mathrm{a}$, the results of simulations with intracellular stimulation are depicted for varying densities of voltage sensitive $\mathrm{Na}^{+}$and $\mathrm{K}^{+}$channels at the lower membrane. The densities are set to respectively $100 \%, 50 \%$ and $0 \%$ of the density at the upper membrane. The amplitude and width of the active response of the electrode potential increases with a decreasing channel density of the lower membrane. The stimulus amplitude had to be increased to $320 \mathrm{pA}$ since the firing threshold is increased due to lower inward $\mathrm{Na}^{+}$current.

Finally, the capacity of the lower membrane is reduced while the lower membrane is passive $(0 \%$ voltage sensitive channel density). In fig. $4 b$, the results are depicted from intracellular stimulation of $320 \mathrm{pA}$, with a membrane capacity of $1,0.1$ and $0.01 \mu F / \mathrm{cm}^{2}$ respectively. The time constant of the membrane potential decreases, due to the changes in lower membrane capacity. As a result, the amplitude of the intracellular action potential increases. The electrode potential resembles the intracellular potential when the lower membrane capacity is strongly reduced. 


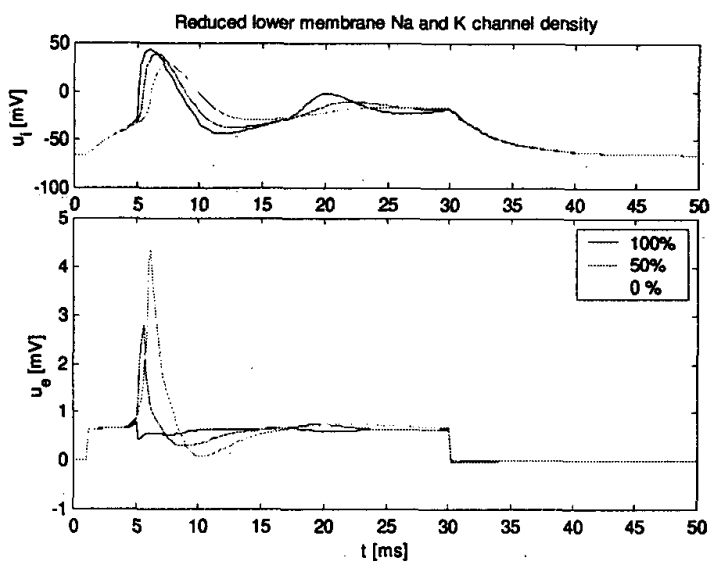

(a)

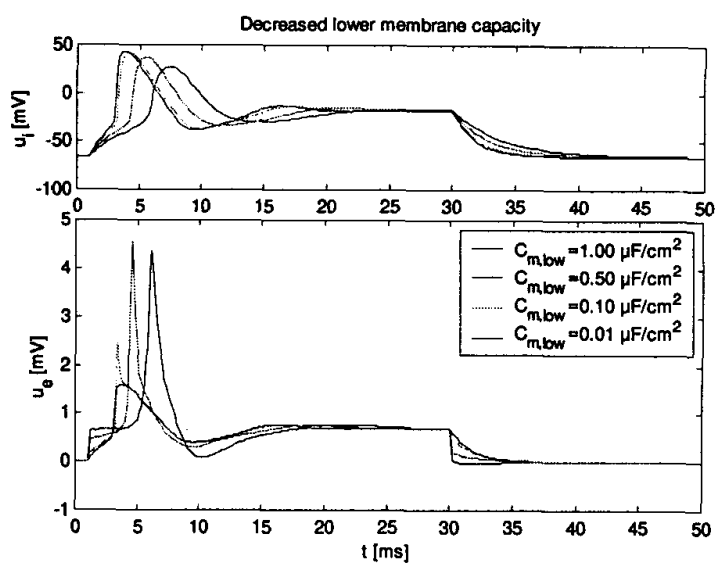

(b)

fig. 4 (a) Intracellular stimulation of a neuron with reduced voltage sensitive $\mathrm{Na}^{+}$and $\mathrm{K}^{+}$channel density at the lower membrane. Extracellular recordings increase in amplitude and width. Also, the electrical behaviour of the neuron is changed.(b) Intracellular stimulation of a neuron with decreased capacity of the lower membrane and without voltage sensitive $\mathrm{Na}$ and $\mathrm{K}$ channels. The recorded extracellular potential resembles the shape of the intracellular action potential when the capacity becomes $0.01 \mu F / \mathrm{cm}^{2}$.

\section{DISCUSSION}

A variety of extracellular potentials can be produced as a response on extracellular or intracellular applied stimuli. These responses are due to currents passing through the sealing gap, which arise from the extracellular electrode or from the neuronal membrane. The 'passive' responses are caused directly by the stimuli, whereas the active responses follow from a (upper or lower) membrane generated current through the lower membrane.

When the electrical properties of the membrane are uniform over the entire neuron, the active response is only due to differences in upper and lower membrane potentials. When this difference is small, as in the case of intracellular stimulation (fig. 3b), the recorded action potential will also be small. However, when this difference is large, as in the case of extracellular stimulation (fig. 3a), large extracellular responses can be obtained. It should be noted that in the latter case the membrane properties also become inhomogeneous, due to the different (in)activation of channels in the upper and lower membrane.

The active response can also be due to altered electrical properties of the lower membrane, even though no difference exists between upper and lower membrane potential. This is the case when the density of voltage sensitive $\mathrm{Na}^{+}$and $\mathrm{K}^{+}$channels is decreased in the lower membrane. When an action potential develops, the inward $\mathrm{Na}^{+}$current density through the lower membrane is lower than that through the upper membrane. The total outward current is controlled by the stimulus and therefore, an outward current density arises through the lower membrane that compensates the difference. In fig. $4 a$, this is mainly a capacitive current and in fig. $4 \mathrm{~b}$ this current becomes more and more ionic, as the capacity of the lower membrane decreases. It should be noted that the total electrical behavior of the neuron is also altered.

The results demonstrate that the model, presented in this paper, is able to compute the local membrane potentials as well as the electrode potential dynamically from extracellular or intracellular current stimuli. Furthermore, the effects of the neuron-electrode geometry, the local membrane properties and the conductivity of the sealing gap are included in the results. Therefore, this model is a valuable tool for studying the effects of sealing on the neuron-electrode contact.

\section{ACKNOWLEDGEMENT}

This work was supported by the BIOMED II EC project, shared cost contract no. BMH4-2723.

\section{REFERENCES}

[1] P. Fromhertz, Eur. Biophys. J., 28, pp.254-258, 1999

[2] W.G. Regehr, J. Pine, C.S. Cohan, M.D. Mischke, D.W. Tank, $J$ Neurosci. Meth., 30, pp. 91-106, 1989.

[3] M. Bove, M. Grattarola, S. Martinoia, G. Verreschi, Bioel. chem, and Bioen., 38, pp. 255-265, 1995.

[4] J.R. Buitenweg, W.L.C. Rutten, E. Marani, Proc. first joint BMES/EMBS conf., ISBN 0-7803-5683-7 (CDROM), 1999. 